\title{
Second-order conditioned taste aversion in rats: Shared modality is not sufficient to promote an association between S2 and S1
}

\author{
DEREK ROBERTSON \\ University of St. Andrews, St. Andrews, Fife, Scotland \\ RICHARD V. KRANE \\ Acadia University, Wolfville, Nova Scotia, Canada \\ and \\ PAUL GARRUD \\ University of St. Andrews, St. Andrews, Fife, Scotland
}

\begin{abstract}
Second-order conditioning (SOC) in pigeons, but not rats, appears to involve an association between the second-order stimulus (S2) and the first-order stimulus (S1). Nairne and Rescorla (1981) suggested it was the use of stimuli from the same modality that promoted an association between $\mathrm{S} 2$ and $\mathrm{S} 1$ in pigeon SOC studies. In support of their hypothesis, they demonstrated that pigeons, like rats, did not form an association between $S 2$ and $S 1$ when these stimuli were from different modalities. In this study, we sought to determine whether rats, like pigeons, would associate S2 with $\mathrm{S} 1$ when these stimuli shared the same modality. Female Lister rats injected with $\mathrm{LiCl}$ after consuming .12M saline solution (S1) showed an aversion to a $15 \%$ sucrose solution (S2) that was subsequently paired with the saline. This was so regardless of whether S2 and S1 had been presented sequentially (Experiment 1) or simultaneously (Experiment 2). Only in Experiment 2 , however, did extinction of the aversion to saline diminish the aversion to sucrose; that is, employing stimuli from the same modality was not a sufficient condition, of itself, to allow rats to associate S2 with S1.
\end{abstract}

Second-order conditioning (SOC), in which one stimulus (S2) elicits a conditioned response (CR) as a result of being paired with another stimulus (S1) whose ability to act as a reinforcer stems from prior association with an unconditioned stimulus (US), has been the subject of several research papers in recent years. Of particular relevance to the experiments reported here are those studies that attempted to establish whether SOC involves an association between $\mathrm{S} 2$ and $\mathrm{S} 1$ such that $\mathrm{S} 2$ elicits whatever response is appropriate to $\mathrm{S} 1$.

The strategy employed by these studies was to manipulate S1 (once SOC had been established to S2) in such a way as to alter the $C R$ elicited by $S 1$ and then test the response to $\mathrm{S} 2$. A change in the $\mathrm{CR}$ to $\mathrm{S} 2$ corresponding to that produced in the $C R$ to $S 1$ would have provided evidence of an association between $\mathrm{S} 2$ and $\mathrm{S} 1$. This procedure produced conflicting results, however, depending upon whether the subjects were rats or pigeons. Experiments using an autoshaping procedure with pigeons

\footnotetext{
This research was supported by Grant B/80/3/1203/1 from the Science and Engineering Research Council of Great Britain to the first author. The first and third authors' mailing address is: Psychological Laboratory, University of St. Andrews, Fife KY16 9JU, Scotland. The second author's mailing address is: Department of Psychology, Acadia University, Wolfville, Nova Scotia, Canada BOP 1 XO.
}

found that extinction of the CR to $S 1$ produced a corresponding change in the strength of the response elicited by S2 (Leyland, 1977; Rashotte, Griffin, \& Sisk, 1977; Rescorla, 1979). In contrast, extinction of the CR to $\mathrm{S} 1$ in conditioned suppression or conditioned activity experiments with rats had no effect on responding to S2 (Holland \& Rescorla, 1975; Rizley \& Rescorla, 1972). Thus, whereas SOC in pigeons appeared to involve an association between $\mathrm{S} 2$ and S1, SOC in rats appeared not to do so.

Nairne and Rescorla (1981) suggested that the discrepant results of SOC experiments with rats and pigeons were due to the nature of the stimuli chosen as $\mathrm{S1}$ and S2: the rat SOC experiments employed lights and tones, whereas pigeon SOC experiments that demonstrated loss of associative strength to $\mathrm{S} 2$ following extinction of the CR to S1 generally employed visual stimuli as S1 and S2 (but see Experiment 4 of Rashotte et al., 1977, for an instance of SOC autoshaping to a visual S2 being decremented by extinction of the CR to an auditory S1). Drawing upon work by Rescorla and Furrow (1977), which demonstrated that similarity between S1 and S2 facilitated the acquisition of SOC, Nairne and Rescorla suggested that the use of stimuli from the same modality may have promoted the formation of an association between $S 2$ and $S 1$ in preference to other potential asso- 
ciations. In support of their hypothesis, Nairne and Rescorla reported that they were unable to replicate the Rashotte et al. (Experiment 4) result; that is, Nairne and Rescorla found that second-order autoshaping to a visual S2 was unaffected by extinction of the CR to an auditory S1. Pigeons, like rats, appeared not to form an association between S2 and S1 when these stimuli were from different modalities.

Confidence in the validity of the Nairne and Rescorla (1981) shared-modality hypothesis, that is, that the discrepant results of previous rat and pigeon SOC experiments in respect of the association entered into by $\mathrm{S} 2$ are attributable to whether or not S2 and S1 shared the same modality, would be strengthened if one could be assured that the failure of Nairne and Rescorla to observe attenuation of autoshaping to a visual S2 following nonreinforced presentations of an auditory $S 1$ did not result from inadequate extinction of responding to $S 1$. Rashotte et al. (1977) administered a series of extinction trials to $\mathrm{S} 1$ until each pigeon reached a criterion of minimal responding to $\mathrm{S} 1$, that is, less than $10 \%$ responding to $S 1$ on two consecutive trials, whereas Nairne and Rescorla administered the same number of extinction trials to all subjects. Experiment 5 of the Nairne and Rescorla paper, in which they administered the most number of extinction trials to an auditory $\mathbf{S} 1$, did observe a corresponding (although statistically nonsignificant) loss of responding to a visual S2. Given the not unreasonable assumption that extinction proceeds at different rates in different individuals, it is possible that $\mathrm{S} 1$ was not fully extinguished in some subjects and that with further extinction trials the loss of responding to S2 would have attained statistical significance. [This possibility was acknowledged by Green \& Schweitzer (1980) in explaining the nonsignificant $(p=.07$, twotailed test) reduction they observed in second-order conditioned keypecking of pigeons to a visual S2 following the administration of a uniform number of nonreinforced presentations of an auditory S1.]

The complementary prediction that follows from the Nairne and Rescorla (1981) shared-modality hypothesis is that rats should form an association between $S 2$ and S1 when these stimuli are from the same modality. Cheatle and Rudy (1978), who employed such a design, found no evidence, however, of an association between S2 and S1. Using an odor-aversion procedure, in which S2 and S1 were both olfactory stimuli, Cheatle and Rudy found that extinguishing the CR to S1 had no effect on responding to S2. Lest it be thought that SOC with a toxicosis first-order US may not involve processes analogous to those underlying SOC experiments using conditioned suppression or conditioned activity procedures, it should be noted that Archer and Sjöden (1982), using a toxicosis first-order US with an exteroceptive $\mathrm{S} 1$ and an interoceptive $S 2$, obtained results identical to those obtained by Rescorla and his associates (Holland \& Rescorla, 1975; Rizley \& Rescorla, 1972) in SOC rat experiments in which $\mathrm{S} 1$ and $\mathrm{S} 2$ were from different modalities.
The Cheatle and Rudy (1978) results, if reliable, challenge the adequacy of the Nairne and Rescorla (1981) hypothesis as an explanation of the different associations entered into by $\mathrm{S} 2$ in rat and pigeon SOC experiments. The Cheatle and Rudy study was atypical, however, in that it employed neonatal rats (aged 7-10 days).

Brain development in the rat is not complete at birth. The period between 5 and 15 days of age has been identified as that during which occurs the most rapid neuroanatomical, neurophysiological, and biochemical changes in the development of the rat brain (Deza \& Eidelberg, 1967; Himwich, 1962). It is not unreasonable to assume that the ability to associate certain classes of events requires the existence of the appropriate neural structures and processes. One might therefore expect to find age-related changes in the ability of the rat to form certain types of association. Indeed, it has been shown that, whereas interoceptive reinforcers [such as food or lithium chloride $(\mathrm{LiCl})]$ can promote associations in 1to 2-day-old rats (Johanson \& Hall, 1979; Rudy \& Cheatle, 1977), exteroceptive reinforcers (such as peripherally applied electric shock) are effective in establishing associative bonds only in rats older than 10 days of age (Haroutunian \& Campbell, 1979). It may be, therefore, that the ability of neonatal rats to form associations that incorporate information about a conditioned reinforcer may occur later in development than the age at which Cheatle and Rudy (1978) tested their rats. Thus, a second purpose of the present study was to test the generality of the Cheatle and Rudy results by using adult rats in a SOC experiment with $\mathrm{LiCl}$ as the firstorder US and novel flavor solutions as $\mathbf{S 1}$ and $\mathbf{S 2}$.

\section{EXPERIMENT 1}

The flavor solutions chosen as $\mathrm{S} 1$ and $\mathrm{S} 2$, and the method of administration, were identical to those used by Bond and DiGuisto (1976) in their successful demonstration of an SOC taste aversion. The present study also followed previous studies in including a group that experienced paired presentation of $S 1$ with the firstorder US, but unpaired presentation of $S 2$ with $S 1$, in order to control for the possibility that responding to $S 2$ in experimental rats might not depend on paired presentation of S2 with S1 (i.e., SOC), but might reflect generalization between $S 1$ and $S 2$ of the $C R$ to $S 1$. A second control group received unpaired presentation of $S 1$ and the first-order US, but paired presentation of S2 with S1. This group was included to ensure that the reinforcing property of $\mathrm{S} 1$ depended on prior pairing with the $\mathrm{LiCl}$ US.

Following a test of conditioning to $S 2$, the CR to S1 was extinguished in half of the experimental rats. A second test of SOC to S2 was then administered. Given that the experimental rats formed an association between $\mathbf{S 2}$ and $S 1$, one would expect rats for which the CR to S1 was extinguished to exhibit less aversion to $S 2$ than 
would experimental rats for which the CR to S1 was not extinguished. To control for any contribution that generalization of extinction might make in attenuating the $C R$ to $S 2$ in those experimental rats for which the CR to $\mathrm{S} 1$ was extinguished, half the control rats were also given a series of nonreinforced exposures to $\mathrm{S} 1$. Similarly, control rats then received a second test presentation of S2.

\section{Method}

Subjects.The subjects were 32 female Lister rats (130-197 g) bred and reared in the Department of Psychology, University of St. Andrews. They were housed in standard laboratory cages with continuous access to dry food pellets. The rats had previously served as subjects in a neophobia experiment in which they were exposed to a $3 \%$ lemon solution and a $1.25 \%$ coffee solution. Assignment to groups in the present study was such as to equate for previous flavor experience. The letters $P$ and $U$ in the group designations indicate whether a group received paired $(P)$ or unpaired (U) presentation of S1 with the first-order US, and of S2 with $S 1$. The letters $E$ and $N$ indicate whether the $C R$ to $S 1$ was extinguished (E) or not (N) between first and second tests of SOC to S2.

Apparatus. Testing took place in four drinking boxes housed in a room separate from that which contained the home cages. These boxes were made of black Perspex $(25 \times 25 \times 40 \mathrm{~cm})$ with an open top and a grid floor. A $50-\mathrm{ml}$ drinking tube, calibrated to $.1 \mathrm{ml}$, was attached to the front wall of each box by a steel clip. The nozzle of the drinking tube protruded into the box via a small hole situated $6 \mathrm{~cm}$ from the bottom and $10 \mathrm{~cm}$ from the side. No food was available in the test box.

Procedure. Access to water was limited to $30 \mathrm{~min}$ per day at the start of the experiment. Commencing at $1200 \mathrm{~h}$, the rats were allowed $10-\mathrm{min}$ access to water in the test box followed by 20 -min access to water in the home cage. The two 10 -min tests of SOC to sucrose and the three $10-\mathrm{min}$ saline presentations likewise commenced at $1200 \mathrm{~h}$. On Days $1-7,4 \mathrm{~h}$ after the daily access to water (i.e., commencing at $1630 \mathrm{~h}$ ), all rats received $1.0 \mathrm{ml}$ of water from a 1-ml syringe placed just inside the mouth.

On Day 8, the first-order conditioning day, all rats received $0.2 \mathrm{ml}$ of $.12 \mathrm{M}$ saline solution. Groups UPE and UPN $(n=4$ in both groups) received the saline $4 \mathrm{~h}$ prior to the daily access to water (commencing at $0800 \mathrm{~h}$ ). Groups PPN, PPE, PUN, and PUE ( $n=6$ in all four groups) received the saline $4 \mathrm{~h}$ after the daily access to water (i.e., commencing at $1630 \mathrm{~h}$ ). The flavor was placed directly into each rat's mouth with the syringe. Immediately after presentation of the saline to Groups PPN, PPE, PUN, and PUE, each rat was injected intraperitoneally with $20 \mathrm{ml} / \mathrm{kg}$ of $.15 \mathrm{M} \mathrm{LiCl}$. The rats in Groups UPN and UPE were also injected with $\mathrm{LiCl}$ at this time. On Day 9, all rats received only the normal 30-min access to water.

On Day 10, the second-order conditioning day, each rat in each group received $0.2 \mathrm{ml}$ of $15 \%(\mathrm{w} / \mathrm{v})$ sucrose solution $4 \mathrm{~h}$ after access to water. Rats in Groups PPN, PPE, UPN, and UPE received $0.2 \mathrm{ml}$ of saline immediately after presentation of the sucrose. Groups PUN and PUE were administered the saline $18 \mathrm{~h}$ after presentation of the sucrose (i.e., $1030 \mathrm{~h}$ on Day 11).

Tests for second-order conditioning were carried out on Day 11. Each rat was placed in the test box for $10 \mathrm{~min}$, and the amount of sucrose consumed during that period was recorded. Upon removal from the test box, the rats were returned to the home cage and given 20-min access to water.

Extinction of first-order conditioning commenced on Day 12. Groups PPE, PUE, and UPE received a 10-min presentation of saline solution in the test box followed by 20 -min access to water in the home cage. This continued until the saline intake of Groups PPE and PUE did not differ from that of Group UPE on 2 consecutive days (i.e., through Day 14). The rats in Groups PPN, PUN, and UPN were allowed to drink water in the test box equivalent in amount to the total saline consumed by Groups PPE, PUE, and UPE, respectively, on these days.

On Day 15, a second test of SOC was administered. All rats were given 10-min access to sucrose in the test box, and the amount consumed during this period was recorded.

\section{Results}

The mean fluid intake of groups on the two 10-min tests of SOC to sucrose and the three 10-min presentations of saline are shown in Table 1. Comparisons of Group PPN with Group PPE, Group PUN with Group PUE, and Group UPN with Group UPE indicated that no difference existed between these subgroups in the amount of sucrose consumed on the first test of conditioning to S2 (all ts $<1.04$ ). Consequently, data for relevant subgroups were pooled.

The groups did not drink equivalent amounts of sucrose on the first test. A one-way analysis of variance (ANOVA) confirmed that these differences were reliable $[F(2,29)=4.54, p<.05]$. A posteriori comparisons with Duncan's multiple range test (DMRT) indicated that Group PPN + PPE drank significantly less sucrose $(6.45 \mathrm{ml})$ than did Group PUN + PUE or Group UPN + UPE $(p<.05)$, and that the latter two groups did not differ from one another in amount of sucrose consumed ( 8.43 and $8.75 \mathrm{ml}$, respectively). These results demonstrate that the experimental rats had acquired an aversion to the sucrose (S2) following a single pairing of sucrose with saline (S1).

The first extinction trial constituted a test of firstorder conditioning to the saline solution; therefore, the data for Trial 1 were analyzed separately. A one-way ANOVA confirmed that the differences in amounts of

Table 1

Results of Experiment 1

\begin{tabular}{|c|c|c|c|c|c|c|c|c|c|c|}
\hline \multirow[b]{2}{*}{ Group } & \multicolumn{2}{|c|}{ Sucrose 1} & \multicolumn{2}{|c|}{ Saline 1} & \multicolumn{2}{|c|}{ Saline 2} & \multicolumn{2}{|c|}{ Saline 3} & \multicolumn{2}{|c|}{ Sucrose 2} \\
\hline & Mean & SE & Mean & $\mathrm{SE}$ & Mean & $\mathrm{SE}$ & Mean & $\mathrm{SE}$ & Mean & $\mathrm{SE}$ \\
\hline $\begin{array}{l}\text { PPE } \\
\text { PPN }\end{array}$ & $\begin{array}{l}6.87 \\
6.03\end{array}$ & $\begin{array}{l}.66 \\
.46\end{array}$ & 7.72 & 1.32 & 11.52 & .81 & 11.00 & .56 & $\begin{array}{l}6.20 \\
6.33\end{array}$ & $\begin{array}{l}.43 \\
.36\end{array}$ \\
\hline PUE & 8.32 & $\begin{array}{r}.40 \\
1.12\end{array}$ & 6.72 & .83 & 11.43 & .89 & 10.53 & .65 & $\begin{array}{l}0.33 \\
8.15\end{array}$ & .65 \\
\hline PUN & 8.55 & .79 & & & & & & & 9.02 & .92 \\
\hline UPE & 8.30 & 1.08 & 11.55 & 1.38 & 12.80 & .96 & 10.90 & .73 & 8.00 & .58 \\
\hline UPN & 9.20 & 1.12 & & & & & & & 7.45 & .55 \\
\hline
\end{tabular}


saline consumed by Group PPE, Group PUE, and Group UPE (see Table 1) were reliable $[F(2,13)=4.04$, $\mathrm{p}<.05]$. A posteriori comparisons with the DMRT indicated that Group PUE and Group PPE did not differ from one another, but that both these groups drank significantly less saline than did Group UPE $(p<.05)$. These results confirm that paired, but not unpaired, presentation of saline with $\mathrm{LiCl}$ conditioned an aversion to the saline.

A $3 \times 2$ ANOVA on the saline consumption of Group UPE, Group PUE, and Group PPE on the second and third extinction trials revealed no effect of groups $[F(2,13)=.52]$ or of trials $[F(1,13)=3.88, p>$ $.05]$, and no groups $\times$ trials interaction $[\mathrm{F}(2,13)=.54]$. The aversion to saline (S1), therefore, appeared to extinguish rapidly, there being no reliable difference between groups in saline consumption after the first extinction trial.

The data of principal interest are the amounts of sucrose consumed by the various groups on the second test of SOC to the sucrose solution (see Table 1). As on the first test of SOC, the rats that experienced paired presentation of saline with $\mathrm{LiCl}$, and of sucrose with saline (viz., Group PPN and Group PPE) drank less sucrose on a 10-min test than did control rats. More importantly, extinguishing the aversion to saline had no apparent effect on responding to the sucrose, as is evident from the comparable amounts of sucrose consumed by Groups PPE and PPN. Thus, second-order conditioning of a taste aversion with flavor solutions as S1 and S2 did not apparently involve an association between $\$ 2$ and $\mathrm{S} 1$. These inferences drawn from an examination of Table 1 were confirmed by a 3 groups $\times 2$ treatments (extinction or nonextinction of CR to S1) ANOVA. This revealed a significant effect due to groups $[F(2,26)$ $=7.73, p<.01]$, but no effect of treatment $[F(1,26)=$ .21] and no groups $\times$ treatments interaction $[F(2,26)=$ $.58]$.

\section{Discussion}

Experiment 1 found no evidence that SOC of a taste aversion in adult rats involves an association between the second- and the first-order CS. This result is in line with that reported by Cheatle and Rudy (1978), who used an odor-aversion procedure with neonatal rats, and indicates that the Cheatle and Rudy result may be obtained with other than neonatal rats.

The failure to obtain evidence of an association between $\mathrm{S} 2$ and $\mathrm{S} 1$ is also consistent with the results of rat SOC experiments that employed conditioned suppression of barpressing (Rizley \& Rescorla, 1972) or a conditioned activity procedure (Holland \& Rescorla, 1975). However, despite employing stimuli from the same modality for $\mathrm{S} 1$ and $\mathrm{S} 2$, the results of Experiment 1 are inconsistent with those of pigeon SOC experiments in which S1 and S2 were from the same modality (Leyland, 1977; Rashotte et al., 1977; Rescorla, 1979).

The failure to obtain evidence of an association between the sucrose and the saline solution in Experi- ment 1 and the failure to find evidence of an association between an olfactory S1 and S2 (Cheatle \& Rudy, 1978) bring into question the adequacy of the Nairne and Rescorla (1981) shared-modality hypothesis as an explanation of the different associations entered into by $\mathrm{S} 2$ in rat and pigeon SOC experiments. At least with respect to SOC experiments that employ a toxicosis first-order US, the available evidence indicates that employing stimuli from the same modality is not a sufficient condition to promote an association between $\mathrm{S} 2$ and $\mathrm{S} 1$ in rats.

\section{EXPERIMENT 2}

In Experiment 1, it was shown that employing stimuli from the same modality is not a sufficient condition to promote an association between $\mathbf{S} 2$ and $\mathrm{S} 1$ in SOC of a taste aversion in rats. Using a sensory preconditioning procedure in which two solutions, A and B, were presented to the rat either simultaneously or sequentially, an aversion conditioned to $\mathrm{B}$, and rats later tested with $\mathrm{A}$, Rescorla (1980) found that rats exhibited a stronger aversion to solution $A$ if $A$ and $B$ had been presented simultaneously rather than sequentially. On the basis of this result, Rescorla suggested that interstimulus associations are especially promoted by simultaneous presentation of the to-be-associated stimuli. Experiment 2, therefore, sought to determine whether simultaneous S2 and $\mathrm{S} 1$ presentation in a SOC taste-aversion procedure would result in an association between $S 2$ and $S 1$ such that subsequent extinction of the CR to S1 would produce a corresponding change in the CR to $\mathrm{S} 2$. Rescorla (1982) reported just such an effect using a conditionedsuppression-of-barpressing procedure with rats when an auditory $\mathrm{S} 1$ that had been paired with a shock US was subsequently presented simultaneously with a visual S2 to establish SOC to S2.

\section{Method}

Apparatus and Procedure. In all unspecified details, the apparatus and procedure were identical to those of Experiment 1 .

Subjects. The subjects were 40 experimentally naive female Lister rats (156-224 g) bred and reared in the Department of Psychology, St. Andrews University. Housing and conditions of maintenance were the same as in Experiment 1.

Procedure. The experimental and preexperimental procedures were identical to those of Experiment 1, with one exception. On the SOC conditioning day, that is, Day 10 , the rats in Group PPE/N and in Group UPE/N received $0.2 \mathrm{ml}$ of $15 \%(\mathrm{w} / \mathrm{v})$ sucrose and $0.2 \mathrm{ml}$ of $.12 \mathrm{M} \mathrm{NaCl}$ delivered directly into the mouth via a syringe $4 \mathrm{~h}$ after the daily access to water (i.e., commencing at $1630 \mathrm{~h}$ ). The sucrose and $\mathrm{NaCl}$ solutions were presented to the rats simultaneously, the solutions being combined in such a way as to maintain the relative concentration of each. The rats in Group PUE/PUN received $0.2 \mathrm{ml}$ of sucrose at $1630 \mathrm{~h}$ on Day 10 and $0.2 \mathrm{ml}$ of $\mathrm{NaCl} 18 \mathrm{~h}$ later (i.e., $1030 \mathrm{~h}$ on Day (1).

\section{Results and Discussion}

The mean fluid intake of groups on the two 10min tests of SOC to sucrose and the three 10-min saline presentations are shown in Table 2. There was no difference between Group PPE and Group PPN, Group PUE and Group PUN, or between Group UPE 
and Group UPN in amount of sucrose consumed on the first test of conditioning to $S 2$ (all ts $<.82$ ). The data for the subgroups within each main treatment condition were therefore pooled. A one-way ANOVA confirmed that the differences in the amounts of sucrose consumed by the various groups were reliable $[F(2,37)=$ $9.20, \mathrm{p}<.001]$. A posteriori comparisons made with the DMRT indicated that Group PPE + PPN drank reliably less sucrose $(6.51 \mathrm{ml})$ than did either Group PUE + PUN or Group UPE + UPN $(\mathrm{p}<.05)$, and that the latter two groups did not differ from one another in amount of sucrose consumed (i.e., 9.20 and $9.24 \mathrm{ml}$, respectively). Thus, a SOC taste aversion to sucrose was demonstrated in Group PPE + PPN.

Group PPE, Group PUE, and Group UPE drank different amounts on the first 10-min saline test (see Table 2). These differences were reliable $[F(2,17)=4.0$, $\mathrm{p}<.05]$. A posteriori comparisons with the DMRT confirmed that both Group PPE and Group PUE, although not differing from each other, drank reliably less saline than did Group UPE $(p<.05)$. This pattern of results is consistent with an aversion's having been acquired to saline in Groups PPE and PUE as a consequence of the saline- $\mathrm{LiCl}$ pairing that these two groups experienced during the first-order conditioning phase of the experiment.

The aversion to saline evidenced by Group PPE and Group PUE extinguished rapidly. A $3 \times 2$ ANOVA on the saline consumption of Group PPE, Group PUE, and Group UPE on the second and third extinction trial indicated that there was no effect attributable to groups $[F(2,17)=.95]$ or to trial $[F(1,17)=.00]$ and no group $X$ trial interaction $[F(2,17)=1.98, \mathrm{p}>.10]$.

The data of principal interest are the results of the second test of conditioning to S2 (see Table 2). Overall, groups that were allowed to drink saline between the first and the second test of aversion to S2 drank more sucrose on the second test than did the corresponding subgroup that was not given intervening experience with saline solution. This trend was confirmed by a $3 \times 2$ ANOVA that indicated no effect of group $[F(2,34)=$ $1.75, \mathrm{p}>.10]$ and no group $\times$ extinction $[\mathrm{F}(2,34)=$ $2.35, \mathrm{p}>.10]$ interaction, but a significant effect due to extinction $[F(1,34)=7.98, p<.01]$. Multiple $t$ tests indicated that there was no reliable difference in sucrose consumption between Groups PUE and PUN [t(34)=
.62] or between Groups UPE and UPN [t(34)=.55]. However, Group PPE drank reliably more sucrose than did Group PPN $[\mathrm{t}(34)=3.47, \mathrm{p}<.01]$. Thus, extinguishing the aversion to saline (S1) in the secondorder conditioning group (Group PPE) produced a corresponding decrease in the aversion to sucrose (S2); that is, simultaneous presentation of sucrose and saline apparently resulted in an association between S2 and S1 in the experimental groups. This result, obtained using an interoceptive first-order US and interoceptive stimuli as S1 and S2, replicates that reported by Rescorla (1982) using an exteroceptive first-order US and exteroceptive stimuli as $\mathbf{S} 1$ and $\mathbf{S} 2$ in second-order conditioning of barpress suppression in rats.

Rescorla and Durlach (1981) suggested that simultaneous presentation of two stimuli might especially promote an interstimulus association either because this mode of presentation maximizes conjoint processing of the separate stimuli in short-term memory (STM), thereby enhancing the opportunity to associate the two stimuli, or because the stimuli are perceived by the organism not as two separate elements that must be associated, but as attributes of a unitary stimulus (cf. Gibson, 1969). There are as yet no data to determine which of these alternative conceptions provides the more accurate description of the processing accorded to simultaneously presented stimuli.

\section{GENERAL DISCUSSION}

Second-order conditioning has been demonstrated with both rats and pigeons. However, this learning appears to depend on different mechanisms in the two species. Whereas pigeons appear to associate the second-order CS (S2) with the first-order CS (S1), rats apparently associate $\mathrm{S} 2$ with the $\mathrm{CR}$ elicited by $\mathrm{S} 1$ (Archer \& Sjoden, 1982; Cheatle \& Rudy, 1978; Holland \& Rescorla, 1975; Leyland, 1977; Rashotte et al., 1977; Rescorla, 1979; Rizley \& Rescorla, 1972).

Recently, Nairne and Rescorla (1981) suggested that the different associations entered into by $\mathbf{S} 2$ in rat and pigeon SOC are attributable to a procedural rather than a species difference. Specifically, they suggested that the use of stimuli from the same modality encourages the formation of an association between $\mathbf{S} 2$ and $\mathrm{S} 1$ (as in pigeon SOC experiments), whereas employing stimuli

Table 2

Results of Experiment 2

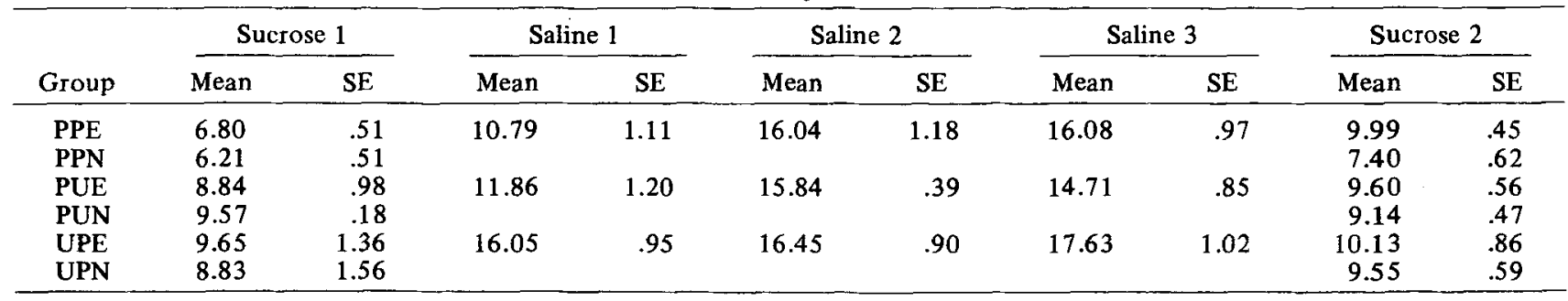


from different modalities (as in rat SOC experiments) allows the CR elicited by $\mathrm{S} 1$ to successfully compete for association with $\mathrm{S} 2$.

Two predictions follow directly from the Nairne and Rescorla (1981) hypothesis. First, pigeons, like rats, should be less likely to associate S2 with S1 when these stimuli are from different modalities. Second, rats, like pigeons, should be more likely to associate S2 with S1 when these stimuli are from the same modality.

Although pigeons have been reported to be capable of forming an association between an auditory $S 1$ and a visual S2, as indicated by loss of responding to S2 following extinction of the CR to S1 (Experiment 4 of Rashotte et al., 1977), Nairne and Rescorla (1981) were unable to replicate this result. We have already suggested that failure to replicate Rashotte et al. (Experiment 4) does not provide compelling evidence in support of the Nairne and Rescorla shared-modality hypothesis because of the possibility that the failure to obtain loss of keypecking to a visual S2 following extinction of responding to an auditory $S 1$ may have resulted from insufficient extinction of the CR to $\mathrm{S} 1$.

With regard to the second prediction of the Nairne and Rescorla (1981) hypothesis, Cheatle and Rudy (1978) had previously demonstrated that rats did not form an association between an olfactory S2 and S1 (as one would have expected them to have done if the Nairne and Rescorla hypothesis were valid) in a SOC odoraversion experiment. Nairne and Rescorla did not comment on the Cheatle and Rudy results, perhaps because the Cheatle and Rudy study was unusual in that it employed neonatal rats and an atypical procedure, thereby occasioning doubts as to the generality of their results. Experiment 1 of the present study, however, using adult rats and gustatory stimuli as $\mathrm{S} 2$ and $\mathrm{S} 1$ in a SOC tasteaversion procedure, confirmed Cheatle and Rudy's observation that extinction of the CR to $S 1$ does not affect responding to $\mathrm{S} 2$ in $\mathrm{SOC}$ with rats in which $\mathrm{S} 2$ and $\mathrm{S} 1$ are from the same modality, that is, that shared modality is not a sufficient condition to generate an association between $\mathbf{S} 2$ and $S 1$ in rat SOC experiments that employ a toxicosis first-order US.

In Experiment 2 of the present study, extinction of the $\mathrm{CR}$ to $\mathrm{S} 1$, following the simultaneous presentation of first- and second-order CSs from the same modality, did produce a corresponding decrease in the magnitude of the CR exhibited toward S2. This result is consistent with the hypothesis that an association was formed between S2 and S1 as a result of their joint presentation, the consequence of this association being that $\mathbf{S} 2$ elicited whatever response was appropriate to $\mathrm{S} 1$. This result, however, is contrary to that which was reported by Archer and Sjoden (1982). Those authors employed an exteroceptive $S 1$ and an interoceptive $S 2$ in a SOC tasteaversion experiment with rats. Although $S 2$ and $S 1$ were presented simultaneously, subsequent extinction of the CR to SI did not affect the strength of aversion exhibited toward S2. Given Archer and Sjoden's failure to obtain evidence that rats form an association between simultaneously presented stimuli from different modalities, Experiment 2, which obtained evidence consistent with rats' having formed an association between two simultaneously presented stimuli from the same modality, lends some support to the Nairne and Rescorla (1981) hypothesis regarding the conditions that favor the formation of an association between $\mathrm{S} 2$ and $\mathrm{S} 1$ in SOC.

Although Experiment 2 illustrates that there are conditions under which the nature of the association underlying SOC with rats mirrors that which underlies SOC with pigeons, there remains the question of why pigeons form an association between $\mathrm{S} 2$ and $\mathrm{S} 1$ when these are from the same modality and are presented sequentially, whereas rats (at least with a toxicosis firstorder US) appear not to do so. One possibility that may be worth considering is that the nature of the first-order US may in part determine the association entered into by S2. In the pigeon SOC studies, S1 signaled the occurrence of food. The occurrence of food, even to a hungry animal, may be a less salient event than is the experience of toxicosis. The CR elicited by a first-order CS (S1) that has been paired with a food US may therefore possess fewer attention-commanding properties than does the CR elicited by a first-order CS (S1) that has been paired with $\mathrm{LiCl}$. Consequently, the former may be less effective than the latter in competing with $\mathrm{S} 1$ for association with a sequentially presented second-order CS (S2). Such ad hoc reasoning requires further experimentation, of course, to assess its validity. Given our present state of knowledge, it would be premature to dismiss the possibility that the different associations formed by $\mathrm{S} 2$ in rat and pigeon SOC experiments that sequentially present first- and second-order CSs from the same modality are the result of species differences in the processing accorded to particular classes of stimuli.

Given the concern about whether the discrepant results of rat and pigeon SOC experiments with respect to the association entered into by $\mathrm{S} 2$ reflect procedural or species differences, it would be of value to determine the nature of the association formed by $\mathrm{S} 2$ in SOC experiments involving other species. Although there is a growing literature on serial and SOC of the rabbit nictitating membrane response (see Kehoe, 1982), this question has not yet been addressed.

In summary, the experiments reported here only partially supported the Nairne and Rescorla (1981) hypothesis regarding the conditions that promote an association between S2 and S1 in SOC. Rats did not form an association between two sequentially presented gustatory stimuli in a SOC taste-aversion procedure (Experiment 1), but did form an association between the same two gustatory stimuli when these were presented simultaneously (Experiment 2). It is not possible to conclude whether the association of S2 with S1 demonstrated in Experiment 2 occurred because simultaneous presentation of the two stimuli maximized conjoint processing of S2 and S1 in STM, thereby allowing S1 to better com- 
pete with the CR elicited by $\mathrm{S} 1$ for association with S2, or whether this mode of presentation led to S2 and S1's being perceived by the rat as attributes of a unitary stimulus. Similarly, it is not yet possible to conclude whether the different associations formed by $\mathbf{S 2}$ in rat and pigeon SOC experiments in which $S 2$ and $S 1$, although from the same modality, are presented sequentially are attributable to procedural or species differences.

\section{REFERENCES}

ArCher, T., \& SJödÉN, P.-O. (1982) Higher-order conditioning and sensory preconditioning of a taste aversion with an exteroceptive CS1. Quarterly Journal of Experimental Psychology, 34B, 1-18.

Bond, N. W., \& DiGuisto, E. L. (1976). One trial higher-order conditioning of a taste aversion. Australian Journal of Psychology, 28 , 53-55.

Cheatle, M. D., \& Rudy, J. W. (1978). Analysis of second-order odor-aversion conditioning in neonatal rats: Implications for Kamin's blocking effect. Journal of Experimental Psychology: Animal Behavior Processes, 4, 237-249.

DEZA, L., \& EIDELBERG, E. (1967). Development of cortical electrical activity in the rat. Experimental Neurology, 17, 425-438.

Gibson, E. J. (1969). Principles of perceptual learning and development. New York: Appleton-Century-Crofts.

Green, L., \& SchweITzer, L. (1980). Second-order conditioning of the pigeon's keypeck using an autoshaping procedure. American Journal of Psychology, 93, 25-39.

Haroutunian, V., \& Campbell, B. A. (1979). Emergence of interoceptive and exteroceptive control of behavior in rats. Science, 205, 927-929.

Himwich, W. A. (1962). Biochemical and neurophysiological development of the brain in the neonatal period. International Review of Neurobiology, 4, 117-158.

Holland, P. C., \& RESCORLA, R. A. (1975). Second-order conditioning with food unconditioned stimulus. Journal of Comparative and Physiological Psychology, 88, 459-467.

Johanson, I. B., \& HaLL, W. G. (1979). Appetitive learning in 1day-old rat pups. Science, 205, 419-421.

KEHOE. E. J. (1982). Conditioning with serial compound stimuli: Theoretical and empirical issues. Experimental Animal Behavior, 1. $30-65$.

LEYLAND, C. M. (1977). Higher-order autoshaping. Quarterly Journal of Experimental Psychology, 29.607-619.

NaIRNE, J. S., \& Rescorla, R. A. (1981). Second-order conditioning with diffuse auditory reinforcers in the pigeon. Learning and Motivation, 12, 65-91.

Rashotte, M. R., Grifin, R. W., \& Sisk, C. L. (1977). Second-order conditioning of the pigeon's keypeck. Animal Learning \& Behavior, 5, 25-38.

Rescorla, R. A. (1979). Aspects of the reinforcer learned in secondorder Pavlovian conditioning. Journal of Experimental Psychology: Animal Behavior Prtocesses, 5. 79-95.

Rescorla, R. A. (1980). Simultaneous and successive associations in sensory preconditioning. Journal of Experimental Psychology: Animal Behavior Processes, 6, 207-216.

RESCORLA, R. A. (1982). Simultaneous second-order conditioning produces S-S learning in conditioned suppression. Joumal of Experimental Psychology: Animal Behavior Processes, 8, 23-32.

Rescorla, R. A., \& DURlach, P. J. (1981). Within-event learning in Pavlovian conditioning. In N. E. Spear \& R. R. Miller (Eds.), Information processing in animals: Memory mechanisms. Hillsdale, $\mathrm{NJ}$ : Erlbaum.

Rescorla, R. A., \& Furrow. D. R. (1977). Stimulus similarity as a determinant of Pavlovian conditioning. Journal of Experimental Psychology: Animal Behavior Processes, 3, 203-215.

Rizley, R. C., \& Rescorla, R. A. (1972). Associations in secondorder conditioning and sensory preconditioning. Journal of Comparative and Physiological Psychology, 81, 1-11.

Rudy, J. W., \& ChEatle, M. D. (1977). Odor-aversion learning in neonatal rats. Science, $198,845-846$.

(Manuscript received March 30, 1983; revision accepted for publication February 17, 1984.) 